\title{
El glicerol como estabilizante de revoques de tierra
}

\author{
Glycerol as earthen coatings stabilizer
}

\section{Resumen}

Autores:

Luis Fernando Guerrero Baca* luisfo1960@yahoo.es Esmeralda Ávila-Boyas* arqavilaboyas80@gmail.com Lilian García-Alonso** lilian_garcia_a@encrym.edu.mx

*Universidad Autónoma Metropolitana-Xochimilco ** Escuela Nacional de Conservación, Restauración y Museografía "Manuel del Castillo Negrete"

Recibido: 12/Mar/2021 Aceptado: 10/Ago/2021

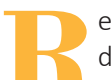

ecientemente ha habido un incremento significativo en la generación de glicerol, que es un subproducto de diversos procesos industriales y que, por sus cualidades aglutinantes, tiene potencial de empleo en la aplicación de revestimientos de tierra para mejorar su resistencia y durabilidad. En este artículo se documenta una serie de análisis comparativos de morteros de tierra natural y otros estabilizados con glicerol, para caracterizar la cohesividad, cantidad de agua de mezclado requerida y resistencia a las afectaciones hídricas por absorción capilar e intemperismo, que son los factores que más afectan a los revoques. Se pudo verificar un incremento en la tenacidad de los morteros estabilizados, una disminución en la cantidad de líquido necesario para mezclar y una adecuada resistencia a la absorción, tanto en laboratorio como en muestras expuestas a la intemperie. El glicerol demostró ser un recurso económica y ecológicamente sostenible para mejorar los revoques de tierra.

Palabras clave: sostenibilidad; bioconstrucción; humedad; revestimientos; arcillas.

\section{Abstract:}

Recently there has been a significant increase in the generation of glycerol, which is a by-product of several industrial processes and which, due to its binding qualities, has potential for use in the application of earth coatings to improve its resistance and durability. This article documents a series of comparative analyzes of natural earth mortars and others stabilized with glycerol that characterize the cohesiveness, amount of mixing water required and resistance to water damage by capillary absorption and weathering, which are the factors that mostly affect plasters. It was possible to verify an increase in the toughness of the stabilized mortars, a decrease in the amount of liquid needed to mix and an adequate resistance to absorption both in the laboratory and in samples exposed to the elements. Glycerol proved to be an economically and ecologically sustainable resource for improving earth plasters.

Keywords: sustainability; bioconstruction; moisture; plasters; clays. 


\section{Introducción}

Dentro de los principios de la bioconstrucción y el diseño ecológico destaca la idea de emplear materiales constructivos de bajo impacto ambiental, que no afecten la salud de los habitantes de los espacios y que, para su implementación, resulten compatibles con el entorno natural, que es su fuente de inspiración (Guerrero, 2019). El uso de la tierra en la edificación ha mostrado ser plenamente sostenible desde el punto de vista económico, ecológico y sociocultural, al haber permanecido por milenios como parte de las culturas constructivas de innumerables sociedades de todo el orbe.

Sin embargo, una de las condiciones que caracterizan a los componentes constructivos hechos de tierra es su interacción con las comunidades que los han realizado. Se trata de sistemas que requieren de la vigilancia de su integridad y, en caso necesario, la realización de acciones de mantenimiento preventivo, puesto que son sensibles a los cambios drásticos de humedad.

Dentro de los componentes arquitectónicos construidos con tierra que requieren mayor cuidado, destacan los revoques porque justamente tienen la función de proteger a las estructuras. Por eso, esta parte de la edificación tradicionalmente ha sido cuidadosamente ejecutada para evitar que, con el paso del tiempo, pueda resultar afectada por la invasión de fauna parásita, hongos, sales minerales o factores atmosféricos como viento, lluvia, granizo o nieve. Un revestimiento de tierra requiere de medios de realización y de substancias coadyuvantes que le permitan enfrentar a los agentes del

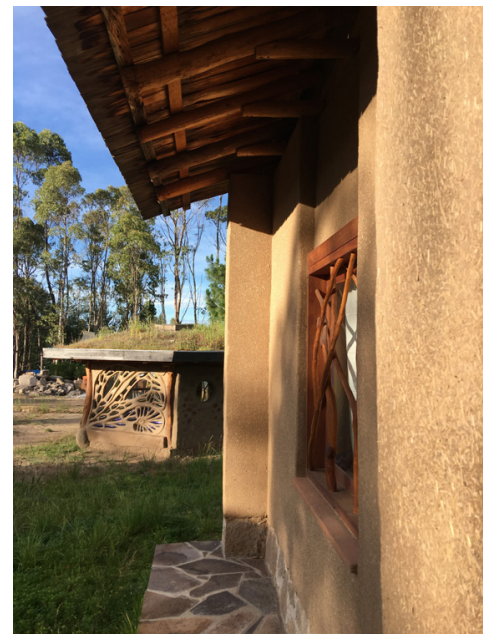

Figura 1: Revoques de tierra estabilizada con paja picada que permanecen a la intemperie sin presentar afectaciones. Proyecto San Isidro, diseñado y construido por la Arq. Alejandra Caballero en Tlaxco, Tlaxcala, México.

Fuente: Elaboración propia (2020) entorno sin que se pierda su permeabilidad, adherencia y potencial de control de las condiciones higrotérmicas de los espacios (Figura 1).

La tierra empleada en los revoques presenta cierta vulnerabilidad al impacto pluvial y a la absorción freática debido a las características higroscópicas de las arcillas, que son la parte esencial de su composición. Para contrarrestar estos agentes de deterioro y dar mayor durabilidad y resistencia a estos componentes, a lo largo de siglos de experimentación las civilizaciones antiguas y las comunidades tradicionales han utilizado diferentes materiales que sirven para mejorar las propiedades de las mezclas. En cada región del planeta se ha probado agregar substancias que abundaban en la naturaleza y que permitían incrementar la cohesividad de los revestimientos, evitar su fisuración al secado y regular su capacidad de absorción de humedad (Minke, 2013).

La tierra como componente constructivo requiere tener condiciones óptimas de humedad durante su transformación y como parte de la arquitectura. La falta o exceso de agua en las mezclas constructivas, y a lo largo de la vida útil de las estructuras, paulatinamente provoca consecuencias indeseables. En este sentido el factor tiempo es crucial. Un edificio puede permanecer por siglos expuesto a la intemperie si de manera cotidiana gana y pierde humedad dentro de un determinado nivel de equilibrio. Pero si se presentan variaciones climatológicas que conducen a la inundación, las arcillas tenderán activarse y separarse de las arenas y limos, haciendo que el sistema pierda su estabilidad. Y en el extremo opuesto, las obras de tierra expuestas por largos periodos de tiempo a soleamiento y viento intensos terminarán por resecarla y la harán deleznable.

Del mismo modo, cuando se realizan mezclas de tierra para elaborar adobes, muros de tapia, de bajareque o revestimientos, si se excede la cantidad de agua o si se trabaja en condiciones de rápida desecación, las estructuras presentarán fisuras por la diferencia en el tiempo de secado entre sus caras expuestas y los sustratos.

Solo por citar un ejemplo relativo al manejo del tiempo en la elaboración de adobes, Vitruvio, el arquitecto romano que vivió a principios de nuestra era, en el tercer capítulo del segundo de sus Diez libros de la arquitectura, indicaba que:

[Los adobes]...se deben hacer en primavera o en otoño, con objeto de que se vayan secando por todas partes de una manera uniforme; en cambio los que se hacen durante el solsticio son defectuosos, porque el sol ardiente seca pronto su corteza, dándoles apariencia de secos, pero luego, cuando efectivamente se han secado, se contraen y, resquebrajándose su superficie, se estropean completamente. Los mejores serán los hechos dos años antes, puesto que pueden secarse preferentemente por su parte interna antes de este tiempo. Con los que se utilizan en fresco y no secos del todo, resulta que el revoque que se les da encima y que adquiere una consistencia rígida que permanece invariable; ellos, en cambio, no pueden conservar la misma rigidez que el enlucido, no se adhieren a él y por la contracción se separan. De suerte que los enlucidos separados de la pared en razón de su escaso espesor, no son capaces de sostenerse por sí mismos y terminan por soltarse, y hasta la propia pared se resquebraja de manera desigual y acaba por estropearse. Por eso en Útica no se permite construir sino con adobe seco, hecho cinco años antes y aceptado por el magistrado (Vitruvio, 1986, pp.40-41) 
De ahí la relevancia del diseño de estrategias de trabajo que garanticen procesos de humidificación y secado controlados de los componentes de tierra, así como de empleo de substancias que permitan regular el tiempo requerido para su adecuado endurecimiento (Kita et. al., 2013)

Dentro de los agregados que históricamente se han empleado para regular la ganancia y pérdida de humedad de la tierra utilizada en los revestimientos se encuentran las grasas de origen animal o vegetal, e hidróxido de calcio, las fibras y los extractos de algunas plantas. Los mucílagos de cactáceas, especialmente los derivados del género Opuntia, se utilizaron desde épocas remotas en Mesoamérica para proteger las superficies arquitectónicas (Torres et. al., 2015). Este material se ha agregado a pinturas y morteros de cal como un retardante del fraguado (Figura 2) que, además, disminuye el porcentaje de contracción de las mezclas y aumenta la fluidez de los morteros empleados para intervenciones de conservación y restauración (Pérez, 2009). Se han logrado resultados similares en estudios realizados en morteros y recubrimientos de tierra en los que se pudo comprobar que el mucílago de la cactácea conocida en México como nopal (Opuntia ficus indica), eleva las cualidades de las mezclas al retardar el secado y permitir la formación de estructuras cristalinas más estables (Avila y Guerrero, 2018).

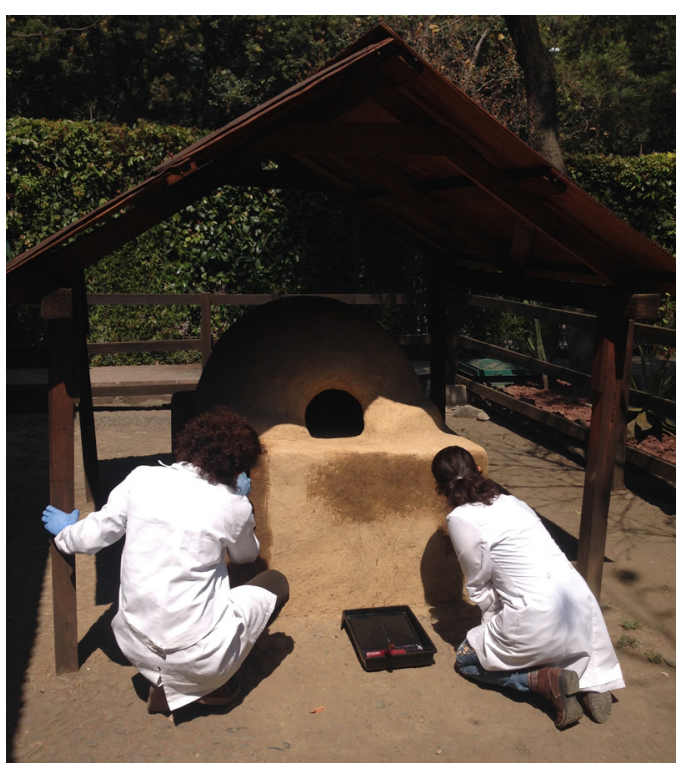

Figura 2: Aplicación de revoques de tierra estabilizada con mucílago de Opuntia durante la restauración en un horno patrimonial en el Museo Nacional de Antropología e Historia de la Ciudad de México.

Fuente: Elaboración propia (2016)

Al buscar alternativas al nopal que pudieran ser más asequibles a nivel internacional, se planteó la alternativa del uso del glicerol -comercialmente conocido como glicerina- que es un alcohol que posee tres grupos hidroxilos $(-\mathrm{OH})$. Se trata de un compuesto líquido a temperatura ambiente, viscoso, incoloro, inodoro y ligeramente dulce. La presencia de los tres grupos hidroxilo le hacen ser sumamente higroscópico, soluble en agua y alcoholes, pero insoluble en hidrocarburos. E glicerol forma parte de las grasas de muchos seres vivos en forma de mono, di o triglicéridos, así como de los fosfoglicéridos que constituyen la membrana celular de animales y vegetales.

Es importante resaltar que actualmente el $70 \%$ de la producción mundial de glicerol se obtiene como subproducto durante la fabricación de biodiesel. Por cada $10 \mathrm{~kg}$ de biodiesel se genera $1 \mathrm{~kg}$ de glicerol (Betancourt et. al., 2016). El biodiesel es un combustible constituido por una mezcla de ésteres monoalquílicos de ácidos grasos, que se obtiene mediante la reacción de transesterificación o alcohólisis de aceites vegetales ricos en triglicéridos, con alcoholes como etanol o metanol, y que, en su síntesis, se extrae glicerol como subproducto (Lafuente, 2017).

Este componente tiene una amplia gama de aplicaciones. Su primer uso conocido data de 1866, en la síntesis del nitroglicerol, componente básico de la nitroglicerina, pero actualmente cumple más de 1500 funciones. El glicerol se utiliza en preparados médicos, farmacéuticos y en cosmética, como un medio para mejorar su suavidad y lubricación. Debido a que es una sustancia higroscópica, se emplea como humectante. También es ampliamente utilizado como laxante y en los jarabes para la tos y expectorantes. Su sabor, ligeramente dulce, unido a su propiedad de no elevar los niveles de azúcar en sangre, hace que sea además muy utilizado como aditivo alimentario. Pero una de sus mayores aplicaciones se da en el ámbito del cuidado personal, pues sirve como emoliente y disolvente de enjuagues bucales, cremas de afeitar, jabones, pastas dentales, productos para la piel y el cabello, entre muchas otras.

Empero, debido al crecimiento acelerado de la producción de biodiesel, y la marcada tendencia a su incremento a nivel mundial, la elevación en los volúmenes de glicerol como subproducto está llegando a convertirse en un problema tecnológico y ecológico. A pesar de su alto potencial de aplicación, se está lejos de emplearlo en su totalidad, con lo que se tienen excedentes que corren el riesgo de afectar al medio ambiente. Si este residuo se arroja indiscriminadamente a los suelos, se modifica su comportamiento y fertilidad porque tiene la capacidad de alterar la organización cristalina y porosidad de la tierra. Sin embargo, esta problemática sobre el medio ambiente puede ser aprovechada como una cualidad, si se propicia su empleo controlado en una gama más amplia de aplicaciones.

En investigaciones realizadas en el sector agrícola, desde 2015 se ha podido demostrar que el uso de dosificaciones reguladas de glicerol como estabilizante edafológico funciona como una materia orgánica que incrementa el crecimiento de las plantas e inmoviliza el nitrógeno añadido como nitrato de amonio, lo cual disminuye el uso de fertilizantes y mitiga la contaminación ambiental (Betancourt et. al., 2016).

A partir de las investigaciones anteriormente citadas, y de las propiedades fisicoquímicas del glicerol, se decidió realizar una serie de experimentos para utilizarlo como un agregado a los recubrimientos de tierra y verificar su comportamiento al estar expuestos a la intemperie. Se partió de la hipótesis de que este compuesto presenta características más adecuadas como añadido en revocos de tierra que substancias más comúnmente utilizadas en 
México, como la cal o el mucílago del nopal (Pérez et. al., 2017). Estos estudios forman parte del proyecto de Investigación Académica titulado "Análisis del tepetate como material para la bioconstrucción y la conservación del patrimonio edificado en Tlaxcala", que fue aprobado por la División de Ciencias y Artes para el Diseño de la Universidad Autónoma Metropolitana Xochimilco (UAM-X), en la Ciudad de México.

\section{Métodos}

La tierra empleada como material constructivo tiene límites de respuesta hídrica muy estables, pero cuando se superan sobrevienen procesos de destrucción difíciles de revertir. Por esta razón, durante décadas se han probado diferentes recursos para la protección superficial de estructuras térreas, muchas de las cuales se han diseñado a partir de la equivocada lógica de la impermeabilización, que se deriva del uso de componentes constructivos de origen industrializado y asociados a la tecnología actual de edificación convencional (Guerrero, 2015)

Como ha sido ampliamente documentado, el uso de substancias impermeabilizantes como los selladores vinílicos o acrílicos, son altamente perjudiciales porque, además de limitar la valiosa cualidad de la tierra como regulador higrotérmico, propician la condensación del vapor de agua y la generación de hongos y microorganismos dañinos para la salud (Mattone et. al., 2016).

La tierra es un material que requiere "transpirar y respirar", es decir, intercambiar aire y vapor de agua con el entorno, lo cual genera un funcionamiento similar a la climatización mecánica. Cuando los espacios presentan una elevada humedad relativa, los revoques de tierra absorben el exceso de vapor de agua, y, si los ambientes están muy secos, los revestimientos dejan salir vapor de agua, con lo que se consigue una confortable estabilidad higrotérmica (García et al., 2020).

El erróneo empleo de recursos impermeables como protección superficial de edificios conduce a la migración desales, la condensación de humedad, el encapsulamiento de líquido en los sustratos, las exfoliaciones y, en casos extremos, al colapso de componentes constructivos por disgregación o disolución.

Para verificar la viabilidad del empleo de recubrimientos estabilizados con glicerol, aplicados tanto sobre elementos constructivos de tierra como en otros materiales, se realizó una serie de experimentos que partieron de la definición de un tipo unificado de suelo que permitiera considerarlo como una constante de investigación. Las variables de trabajo fueron el tiempo, la humedad y la cohesividad de las mezclas usadas como probetas expuestas a condiciones cambiantes. Como referente para evaluar las propiedades del glicerol se utilizó la tierra mezclada solamente con agua, con base en la experiencia desarrollada en años anteriores con relación al estudio del comportamiento del mucílago de Opuntia Ficus en revestimientos de tierra (Guerrero y Ávila, 2019).
La investigación fue realizada en diversas etapas en el Laboratorio de Tecnologías Tradicionales de la Escuela Nacional de Conservación Restauración y Museografía (ENCRYM-INAH), así como en el Taller de Construcción con Tierra de la UAM-X en la Ciudad de México. La tierra que se utilizó para la elaboración de las muestras fue extraída de la zona de Tepecoacuilco, en el estado de Guerrero, al sur de la República Mexicana. El material natural fue compensado con diferentes volúmenes de arena hasta conseguir una mezcla que tuviera una adherencia adecuada a los sustratos, pero que al secarse no presentara retracciones y fisuras, que son condiciones fundamentales que se esperan de cualquier revestimiento. Como se ha demostrado en diversos estudios (Cerro y Baruch, 2011; Minke, 2013; Mattone et. al. 2016) los recubrimientos arenosos son recomendables para propiciar la evapotranspiración de las superficies y evitar la condensación que puede generar deterioros y la aparición de microrganismos y hongos.

Los datos proporcionados por el laboratorio "Geotécnica y Suelos" indicaron que la mezcla final que cumplía con los requerimientos antes citados, contenía un 81\% de arena y $19 \%$ de arcilla y limos, de manera que su Límite Líquido era de 23,4; su Límite Plástico de 15,0 y su Índice de Plasticidad de 8,4, por lo que correspondía, de acuerdo al Sistema Unificado de Clasificación de Suelos (SUCS), como una tierra tipo "SC", o sea, una "Arena Arcillolimosa".

Para formar la solución glicerol-agua se tomó como referencia la dosificación derivada de los trabajos realizados en investigaciones anteriores (Ávila y Guerrero, 2018; Guerrero y Ávila, 2019) en los que se empleó 1\% de mucílago de nopal como estabilizante, por lo que se procedió a agregar $10 \mathrm{ml}$ de glicerina vegetal USP en cada litro de agua.

El estudio continuó con la evaluación comparativa para medir la cohesividad de la mezcla. A falta de estándares mexicanos para sistemas constructivos de tierra se tomó como referencia el procedimiento empleado desde los años ochenta en la Universidad de Kassel (Minke, 2005). El ensayo consistió en la elaboración de mezclas de tierra con agua y de tierra con la solución de glicerol con la que se conformaron series de barras cilíndricas de $20 \mathrm{~cm}$ de largo por $1,5 \mathrm{~cm}$ de diámetro, que se colocaron paulatinamente sobre una hoja de papel situada en la orilla de una mesa. Posteriormente el papel era jalado fuera de la mesa de manera que quedara colgando la barra hasta que finalmente se rompiera (Figura 3).

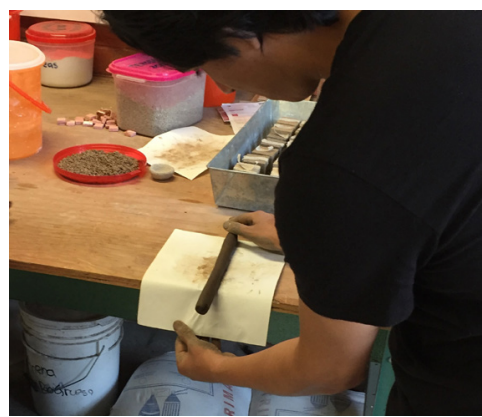

Figura 3: Ensayo de cohesividad realizado a barras de tierra en estado plástico Fuente: Elaboración propia (2018) 
Cada fracción desprendida de la barra se colocaba sobre la mesa para ser medida. En este ensayo se busca evaluar la longitud de las porciones en las que se rompe cada barra, la cual está correlacionada con la cohesividad de material. La longitud mayor significa que la tierra está mejor cohesionada, mientras que, en el polo opuesto, fracciones cortas denotan tierras con poca consistencia. Como referencia se ha documentado que para revoques resultan apropiadas porciones de 5 a $8 \mathrm{~cm}$ (Minke, 2013)

En el proceso de desarrollo de las pruebas de cohesividad se observó que se requirió agregar menor cantidad de solución glicerol-agua para las barras, que el volumen empleado en las mezclas testigo a las que solo se agregó agua. También se notó que la consistencia y plasticidad de la mezcla con glicerol mejoraba notablemente. Por esta razón se desarrolló una serie de pruebas adicionales que permitieran verificar el punto exacto de líquido requerido para que la mezcla alcanzara su condición óptima de trabajabilidad, comparándose siempre el uso del agua sola con la solución de glicerol.

Posteriormente, se realizaron mezclas de tierra-agua y tierra-solución de glicerol formando tabletas de $10 \mathrm{~cm}$ $\times 10 \mathrm{~cm} \times 0,5 \mathrm{~cm}$ vaciadas en moldes de madera. Estas tabletas se utilizaron para los ensayos de absorción hídrica superficial con base en la norma española UNEEN-16302, a falta de estándares nacionales para ese tipo de evaluaciones. El ensayo consiste en tomar el tiempo que tarda en penetrar una cantidad definida de agua en una superficie determinada del material a evaluar.

El instrumento para realizar los ensayos es el tubo de Karsten. Se sella la unión del tubo y la superficie de contacto con una masilla impermeable para evitar fugas y concentrar el flujo del agua, el cual ejerce una presión en la "superficie de 961.38Pa, la cual corresponde a la acción de gotas de lluvia golpeando a la pared con una velocidad del viento estática de $140 \mathrm{~km} / \mathrm{h}$ perpendicular a la superficie" (Pérez, 2016, p. 75).

El estudio posterior consistió en la evaluación comparativa de absorción capilar para la que se realizaron probetas cúbicas de $5 \mathrm{~cm} \times 5 \mathrm{~cm} \times 5 \mathrm{~cm}$, correspondientes a tierraagua y tierra-glicerol, que se secaron por un periodo de 28 días a temperatura y humedad estables. Se tomó como referencia la Norma Italiana (Normal 11/85, 1985), que consiste en colocar cada muestra sobre una tela saturada de agua en una charola durante periodos de 30 segundos para su posterior pesado en una báscula de precisión. Este proceso de absorción y pesado se repite hasta que la muestra comience a perder material por disgregación.

Para la última evaluación se aplicaron recubrimientos de $20 \mathrm{~cm} \times 20 \mathrm{~cm} \times 0,2 \mathrm{~mm}$ sobre muros de tierra $y$ bloques de cemento, con el fin de evaluar la adherencia y comportamiento al dejarlas a la intemperie ante la Iluvia directa. Es importante resaltar que, durante la elaboración de las probetas cúbicas, las tabletas y los revoques se registró un fenómeno de notable relevancia como explicación del papel que juega el glicerol en las mezclas de tierra. El tiempo de secado inicial de los tres tipos de componentes fue muy superior cuando estaban estabilizados, en comparación con los elaborados con tierra y agua. Como se detallará en las conclusiones, entre más lento sea el secado de los componentes realizados con tierra, mejor será su organización cristalina, lo que incrementa su resistencia mecánica y durabilidad.

Mientras que los revoques de tierra con agua ya estaban prácticamente secos, las probetas realizadas con glicerol mostraban un color obscuro que evidenciaba la presencia de humedad, la cual permaneció durante dos horas más. En el caso de las tabletas se realizaron evaluaciones del peso durante el secado inicial a temperatura y humedad ambiental a fin de documentar que las primeras tenían peso constante aproximadamente a las 2 horas con 15 minutos, pero las que contenían glicerol tardaron casi seis horas. El mismo fenómeno se presentó al realizar las series de bloques cúbicos para las pruebas de absorción capilar. Los cubos mezclados con agua se secaron a pleno sol en poco más de tres horas, mientras que las de glicerol tardaron casi un día en perder su color obscuro (Figura 4)

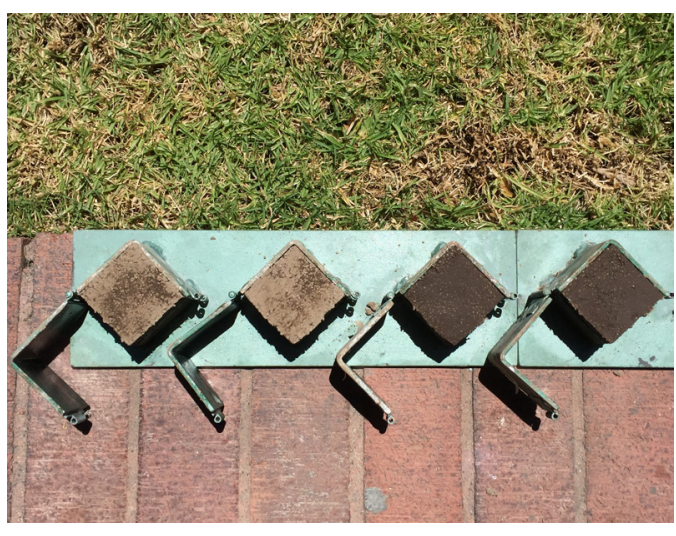

Figura 4: Dos probetas cúbicas realizadas con agua (izquierda) simultáneamente a las mezcladas con la solución de glicerol (derecha), después de tres horas de secado

Fuente: Elaboración propia (2018)

\section{Resultados}

La prueba de cohesividad sirvió para determinar la consistencia de la mezcla y la cantidad de agua requerida para alcanzar la condición óptima de trabajabilidad. Se observó que las fracciones correspondientes a las barras realizadas con agua tuvieron en promedio un punto de ruptura a los $7,5 \mathrm{~cm}$. Sin embargo, las barras que contenían glicerol se rompieron a los $8,5 \mathrm{~cm}$.

Con respecto a la cantidad de agua requerida para la obtención de una mezcla adecuadamente consistente, que permitiera un amasado uniforme, se observó que un volumen de tierra de $700 \mathrm{ml}$ mezclado con agua demandó $170 \mathrm{ml}$, mientras que el mismo volumen de tierra requirió solamente $125 \mathrm{ml}$ de glicerol. Este dato traducido a porcentaje determina que la solución de glicerol reduce la cantidad requerida de líquido de mezclado en un $28 \%$.

Para el ensayo de absorción superficial de humedad con el tubo Karsten, la mezcla que se hizo solo con tierra y agua manifiesta una notoria absorción hídrica, mientras 
que la mezcla con glicerol absorbió solo una tercera parte. La probeta realizada con agua pesaba inicialmente $74,2 \mathrm{~g}$ y después de cuatro minutos $75,3 \mathrm{~g}$. Por su parte la probeta estabilizada pesaba al principio del experimento $76,8 \mathrm{~g}$ y finalmente $77 \mathrm{~g}$. Eso se traduce en un incremento de $6 \mathrm{~g}$ en el primer caso $(1,48 \%)$ y de $2 \mathrm{~g}$ en el segundo $(0,26 \%)$

Como complemento al ensayo de absorción superficial se realizó una prueba de liberación de humedad que se sustenta en la medición del tiempo de secado total de las piezas después de haber sido humedecidas mediante e tubo Karsten. Las tabletas de tierra analizadas y pesadas en su nivel de saturación a los 4 minutos se dejaron en un espacio con condiciones controladas a $25^{\circ} \mathrm{C}$ de temperatura y con una humedad relativa del $66 \%$, sin presencia de viento. El ensayo consistió en pesar cada pieza por periodos de 30 minutos hasta que recuperaran su peso inicial, lo cual era indicio de la liberación total de agua que había sido absorbida.

Con este ensayo se documentó que la mezcla de tierra con agua se secó por completo a los 270 minutos mientras que la tableta de tierra estabilizada con glicerol lo hizo a los 150 minutos.

Posteriormente, la prueba de absorción capilar se llevó a cabo registrando y tabulando el incremento en la masa de probetas cúbicas de $5 \mathrm{~cm} \times 5 \mathrm{~cm} \times 5 \mathrm{~cm}$ humedecidas y pesadas cada treinta segundos durante un lapso total de ocho minutos (Figura 5).

Al igual que en el caso de la prueba de las tabletas con el tubo Karsten, la mezcla solo de agua que pesaba originalmente 50,7g al final alcanzó los 55,3g. Es decir que incrementó su peso en $4.6 \mathrm{~g}$ equivalentes al 9,07\% de su peso inicial.

Por su parte, los cubos hechos con glicerol pesaban originalmente $57,2 \mathrm{~g}$ y al final $60,7 \mathrm{~g}$, lo que se traduce en un incremento de $3,5 \mathrm{~g}$, equivalentes al 6,11\%. Finalmente, los revoques que fueron aplicados y posteriormente sometidos a condiciones de lluvia, se realizaron en secciones de $20 \mathrm{~cm} \times 20 \mathrm{~cm} \times 0,2 \mathrm{~cm}$ de espesor. Se colocaron inicialmente a mano y luego se alisaron con una llana metálica para obtener superficies uniformes. Se dejaron a la intemperie las mezclas de tierra-agua y tierra-solución de glicerol, y su comportamiento se fue monitoreando a lo largo de 8 meses.

Durante la colocación de los revoques se comprobó que, al igual que en la elaboración de las probetas cúbicas, las mezclas que se estabilizaron con glicerol tardaron cerca de tres horas más en secar que las de tierra con agua. Cabe mencionar que estas muestras de recubrimiento se aplicaron sobre diferentes tipos de sustratos para verificar su trabajabilidad, adherencia y compatibilidad. Se colocaron sobre ladrillo rojo bloques de cemento y adobes, pero el resultado en todos los casos fue similar.

Después de la intensa temporada de lluvias se observó que el recubrimiento de tierra-agua de $20 \times 20 \mathrm{~cm}$ tuvo en promedio una pérdida cercana al $80 \%$ mientras que el realizado con glicerol se mantiene con un grado de deterioro notablemente menor, teniendo una pérdida superficial de aproximadamente $10 \%$.

\section{Conclusiones}

Los resultados obtenidos en todas las pruebas que se documentan en el presente artículo permiten reconocer que, a pesar de la relativamente baja cantidad de glicerol que se empleó para realizar las mezclas probadas, el papel que juega esta substancia es muy positivo.

En primer lugar, se observó que durante la realización inicial de morteros en los que se emplea solución de glicerol para hacer probetas cúbicas, tabletas y revoques, se consigue un secado en promedio $260 \%$ más lento del componente constructivo, en comparación con las mezclas que se hicieron solo con agua.

Como se sabe a partir de diversas tradiciones constructivas con tierra, cuando un elemento de este material pierde su humedad de moldeado con demasiada velocidad, se suelen generar procesos de retracción en sus superficies, que se manifiestan en agrietamientos y se traducen en la reducción de su resistencia y durabilidad (Stazi et. al., 2016).

Si durante la construcción de elementos de tierra se cuida que el endurecimiento de las mezclas ocurra

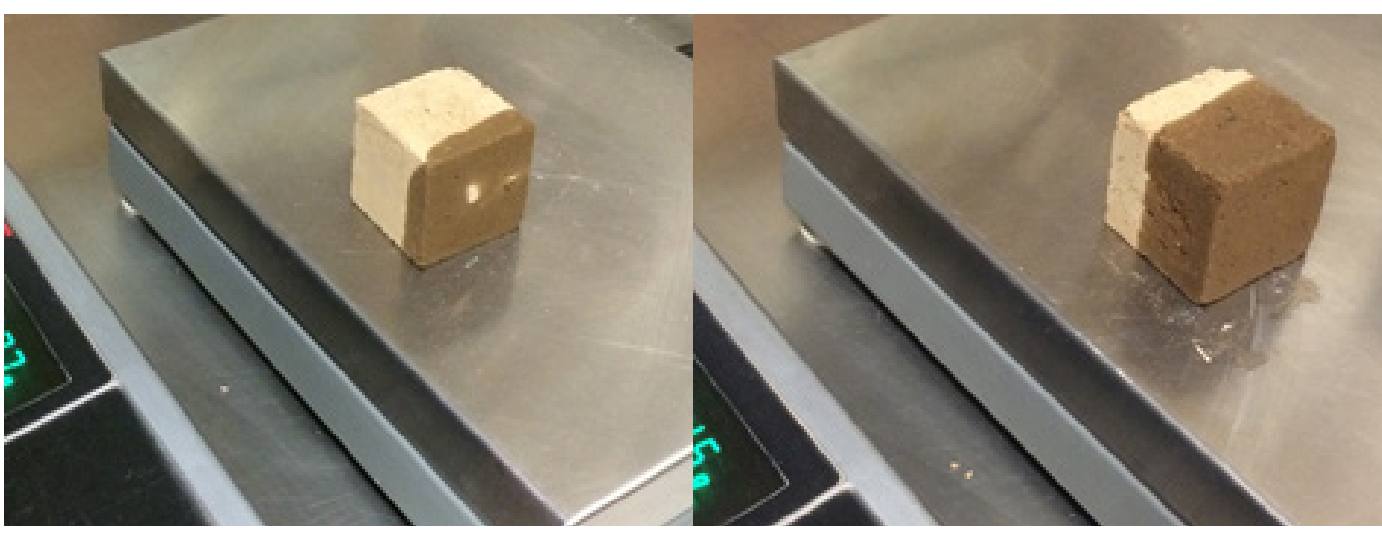

Figura 5: Registro del peso de las probetas cúbicas en periodos controlados, según la Norma Italiana "Normal 11-85" Fuente: Elaboración propia (2018) 
paulatinamente, se consigue una mejor organización cristalina al interior del material, sea un adobe, un mortero o un revoque. Este efecto posibilita la obtención de componentes edilicios más homogéneos y densos en su interior, lo que consecuentemente les confiere mayor resistencia mecánica e hídrica (Guerrero y Ávila, 2019).

En segundo lugar, la prueba de cohesividad puso de manifiesto el incremento en esta propiedad en el orden del $13,33 \%$ al haber pasado la ruptura promedio de las barras de los 7,5cm en la formulación con agua, a los $8,5 \mathrm{~cm}$ al usar la solución de glicerina. La cohesividad es una cualidad muy relevante en lo que concierne a la aplicación de revestimientos pues, al ser más dúctiles, pueden comprimirse mejor, con lo que se obtienen superficies más densas, resistentes y mejor adheridas a los sustratos (Mattone et. al., 2017).

Paralelamente, se comprobó que es posible alcanzar el nivel óptimo de ductilidad de la tierra con $28 \%$ menos cantidad de líquido. Esta aportación resulta también de alto valor ecológico porque implica un notable ahorro de líquido de mezclado durante el desarrollo de los morteros de tierra.

Con respecto a las pruebas de absorción capilar y de secado, los resultados fueron igualmente prometedores. El uso del glicerol retarda el tiempo de absorción capilar en casi un $300 \%$ durante un mismo periodo, lo cual significa que es posible obtener revestimientos altamente resistentes a la humedad.

En lo que concierne al tiempo de desorción del agua absorbida superficialmente o por capilaridad, el resultado fue igualmente destacable pues las mezclas hechas con glicerol se secan $55,5 \%$ veces más rápido. Este dato permite suponer que el agua se inserta entre las micelas de las arcillas, y causa un tiempo de secado muy prolongado mientras que el glicerol les brinda una especie de protección hidrofugante que propicia una rápida evaporación.

Finalmente, el efecto de la lluvia directa es más difícil de cuantificar, pero resulta evidente a partir de las series de fotografías que se tomaron durante el tiempo que duró la prueba, y que, aunque concluyó a los 8 meses de haberse iniciado, el recubrimiento realizado solamente con agua se ha perdido por completo mientras que el de glicerol se mantiene a más de un año de haberse aplicado (Figura 6).

Las pruebas reseñadas en el presente texto demostraron que el empleo de soluciones de glicerol (con solamente
$1 \%$ de concentración) como parte del líquido de amasado de morteros de tierra aplicados como revestimientos, permite retardar su secado con lo que se consigue tener superficies más homogéneas, densas y resistentes al impacto de diversos agentes de deterioro. Estos revestimientos, una vez aplicados y endurecidos, tienen un comportamiento parcialmente hidrofugante, haciendo menos vulnerables a las arcillas al frenar e ingreso del agua entre sus micelas. Con ello se retiene la humedad por menos tiempo, evitándose cambios de volumen y posterior retracción y disgregación (Guerrero, 2016).

Asimismo, las cantidades parciales que se absorben con la tierra cohesionada con glicerol son mucho menores, lo que garantiza la estabilidad de su aplicación como recubrimiento porque, sin convertirse en un componente impermeable, se regula la absorción y desorción del agua. Un ingreso gradual del agua puede ser gestionado adecuadamente por el sistema completo, pero esto no es posible con un ingreso repentino de líquido que puede disgregar los componentes de la tierra.

El uso del glicerol como estabilizante puede suponer mejores características en mezclas para revestimientos de tierra aplicados a obras nuevas, pero, sobre todo, como superficies protectoras y de sacrificio en una amplia gama de estructuras patrimoniales. De este modo se consigue aprovechar la sobreproducción de una substancia potencialmente contaminante, con lo que se contribuye al manejo sostenible de los recursos.

El aprovechamiento del glicerol como componente de estabilización de materiales constructivos de tierra, resultará altamente positivo tanto en el diseño contemporáneo como en la conservación sostenible del patrimonio cultural, desde el punto de vista económico, ecológico y socio cultural.

\section{Recomendaciones}

Ante la pérdida de información sobre los procedimientos y substancias empleadas en la antigüedad para realizar recubrimientos de tierra que sorprenden por su calidad (Figura 7), es necesario desarrollar experimentos que ayuden a explicar su comportamiento. Es recomendable establecer protocolos que permitan analizar de manera unitaria las diferentes variables que se relacionan con la adherencia, porosidad, relación hídrica y resistencia a la intemperie de los revoques porque, aunque se sabe que todas interactúan, cada una juega un papel específico en la protección superficial.

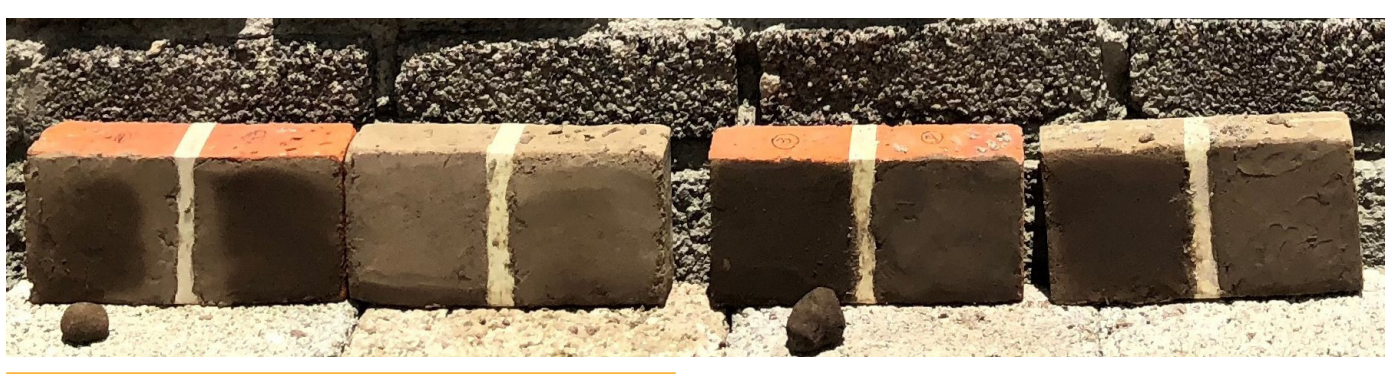

Figura 6: Evaluación de los revestimientos aplicados sobre diferentes tipos de bloques y dejados a la intemperie Fuente: Elaboración propia(2018) 


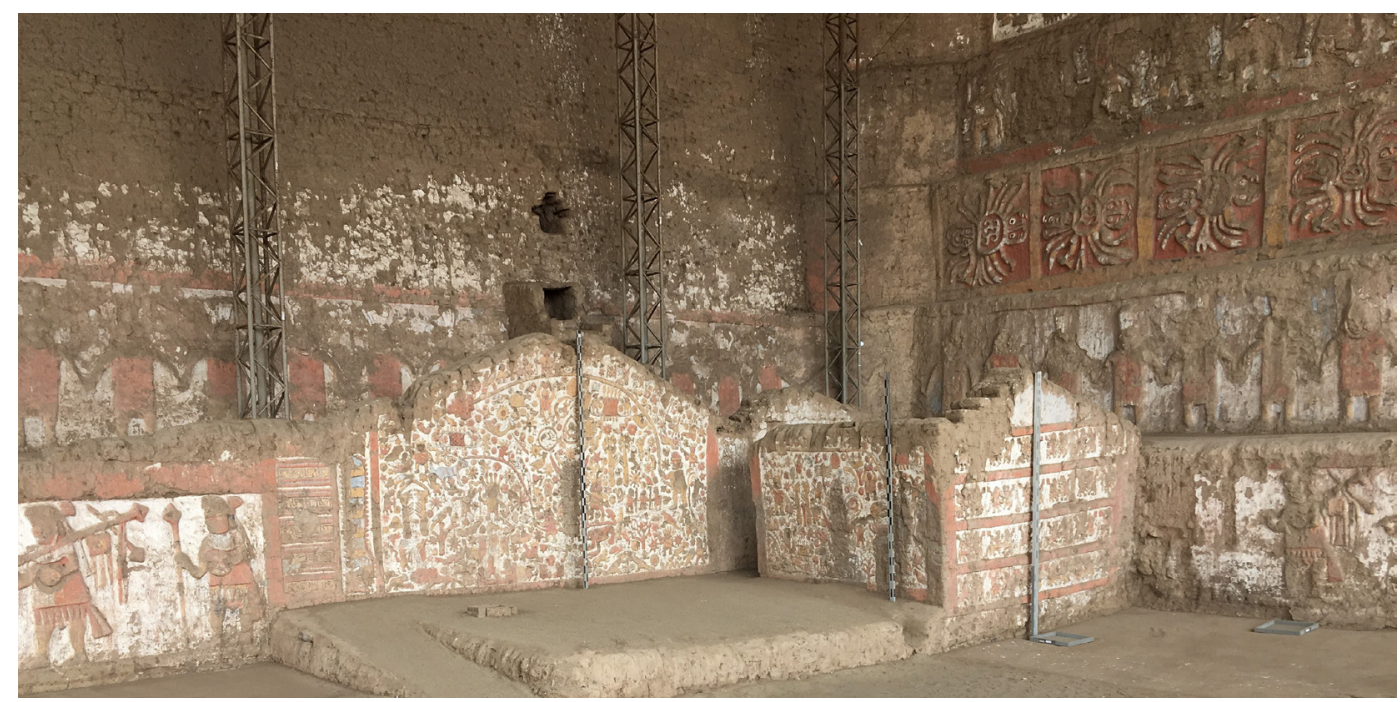

Figura 7: Los revoques realizados con tierra en las Huacas de Moche, Trujillo, Perú, se conservan con alto grado de integridad, a pesar de tener más de mil años de existencia

Fuente: Propia (2018)

Resulta imperativo insistir en la modificación de la concepción de los revoques en la arquitectura de tierra. La influencia de la construcción contemporánea y el uso de revestimientos industrializados han hecho perder de vista la relevancia de la evapotranspiración de los sustratos tradicionales. Se tiende a pensar que el agua en las edificaciones es un enemigo a vencer, o por lo menos a evitar, por lo que normalmente se promueve el uso de protecciones impermeables.

Empero, ese argumento solo aplica para sistemas constructivos que incluyen cemento y acero, puesto que la humedad les provoca daños irreversibles. En los sistemas históricos y vernáculos edificados con materiales porosos, el intercambio cotidiano de vapor de agua con el medio ambiente garantiza su permanencia y el mejoramiento en las condiciones de higiene de los espacios. Las estructuras de tierra se ven afectadas tanto por el exceso como por la falta de agua, de manera que esta debe ser adecuadamente gestionada.

Por otra parte, un campo que abre notables posibilidades de avance en la investigación sobre materiales térreos se deriva del papel del tiempo. Las mezclas que se realizan, reposan, aplican y dejan endurecer con lentitud tienen resultados mucho más estables y duraderos que los que se efectúan velozmente. Por desgracia, se trata de factores que no son evidentes en los edificios históricos pues no se cuenta con medios para verificar la manera en que se procedió para su elaboración en la antigüedad.

Es crucial dejar de ver a los edificios del pasado solo como objetos, para empezar a analizarlos como procesos. La explicación de la sostenibilidad de la arquitectura histórica y tradicional, que ha permitido que permanezca hasta nuestros días, no radica en la materia que contienen sino en la manera en que esta fue transformada, el tiempo que sus autores dedicaron a cada etapa de su elaboración y, sobre todo, las actividades de mantenimiento y conservación preventiva que siempre fundamentaron su cultura constructiva.
Cómo citar este artículo/How to cite this article: Guerrero Baca, L., Ávila-Boyas, E. y García-Alonso, L. (2022). El glicerol como estabilizante de revoques de tierra. Estoa. Revista de la Facultad de Arquitectura y Urbanismo de la Universidad de Cuenca, 11(21), 59-67. https://doi.org/10.18537/est.v011. n021.a05 


\section{Referencias bibliográficas}

Ávila, E. y Guerrero, L. (2018). El mucílago de Opuntia Ficus como estabilizante en recubrimientos de tierra. En Memorias del SIACOT 2018 (pp.115-126). PROTERRA.

Betancourt, C., de Melo Prado, R., Castellanos, L. y Silva, C. (2016). Características de la glicerina generada en la producción de biodiesel, aplicaciones generales y su uso en el suelo. Ediciones INCA, Instituto Nacional de Ciencias Agrícolas.

Cerro, M. y Baruch, T. (2011). Enduits terre \& leur décor mode d'emploi. Eytolles.

García, J. R., Guerrero, L. y Morales, G. (2020). Análisis y evaluación del comportamiento térmico de un sistema bioconstructivo de tierra cruda aplicado en prototipos experimentales. En J. De Hoyos, et. al. (Comp.). Bioconstrucción para la vivienda, pensamientos y técnicas (pp.73-92). Universidad Autónoma del Estado de México.

Guerrero, L. (2015). Recubrimientos de tierra compactada para la conservación del patrimonio arqueológico de México y el Salvador. En Memorias del SIACOT 2015 (pp. 233-244). PROTERRA.

Guerrero, L. (2016). El papel de la humedad y la compactación en la elaboración de recubrimientos de tierra. Construcción con tierra, (7), 11-22.

Guerrero, L. (2019). Introducción en L. Guerrero, (Comp.) Bioconstrucción a detalle (pp. 5-11). IBOMEX-Carteles Editores.

Guerrero L. y Ávila, E. (2019). Pañetes de tierra estabilizada con nopal. Revista Nodo, 14(27), 53-62.

Kita, Y., Daneels, A. y Romo De Vivar, A. (2013). Chemica analysis to identify organic compounds in pre-Colombian monumental earthen architecture. TOJSAT, 3 (1), 39-45.

Lafuente, G. (2017). Glicerol: Sintesis y Aplicaciones UNED Facultad de Ciencias.

Mattone, M., Ibnoussina, M., Rescic, S., Fratini, F. Magrini, D., Mecchi, A. M., y Nocairi, M. (2016). Stabilization of earthen plasters: Exchange of knowledge and experiences between Italy and Morocco. Journal of Materials and Environmental Science, 7 (10), 3647-3655.

Mattone, M., Rescic, S., Fratini, F., y Manganelli, R. (2017). Experimentation of Earth-Gypsum Plasters for the Conservation of Earthen Constructions. International Journal of Architectural Heritage Conservation, Analysis, and Restoration, 11(6), 763-772.

Minke, G. (2005). Manual de construcción en tierra. Fin de Siglo.

Minke, G. (2013). Revoques de barro, mezclas, aplicaciones y tratamientos. Icaria editorial.

Normal 11/85 (1985). Assorbimento d'acqua per capillarità - Coeficiente di assorbimento capillare. CNRICR.
Pérez, A., Guerrero, L., González, J. y Prado, R. (2017). Nopal mucilage as hydration agent for quicklime extraction methods, Ge-conservacion, (11), 189-195.

Pérez, N. (2009). Formulación de un mortero de inyección con mucilago de nopal para restauración de pintura mural. [tesis de maestría, Instituto Tecnológico y de Estudios Superiores de Occidente].

Pérez, N. (2016). Los adobes arqueológicos de la Gran pirámide de Cholula. [tesis doctoral, UNAM].

Stazi, F., Nacci, A., Tittarelli, F., Pasqualini, E., y Munafò P. (2016). An experimental study on earthen plasters for earthen building protection: The effect of different admixtures and surface treatments. Journal of Cultural Heritage, (17), 27-41.

Torres, P., Cruz, S., Flores, S., Peña, N., Fernández, S., Rodríguez, M. y Cruz, A. (2015). La baba y el mucílago de nopal, una alternativa natural para la conservación de acabados arquitectónicos de tierra. Revista Interdisciplinaria, INAH, (99), 93-114.

UNE-EN-16302 (2016). Conservación del patrimonio cultural. Métodos de ensayo. Medición de la absorción de aqua por el método de la pipeta. Aenor. https:// www.une.org/encuentra-tu-norma/busca-tu-norma/ norma?c=N0056787

Vitruvio, M. L. (1986). Los diez libros de la arquitectura. Iberia. 